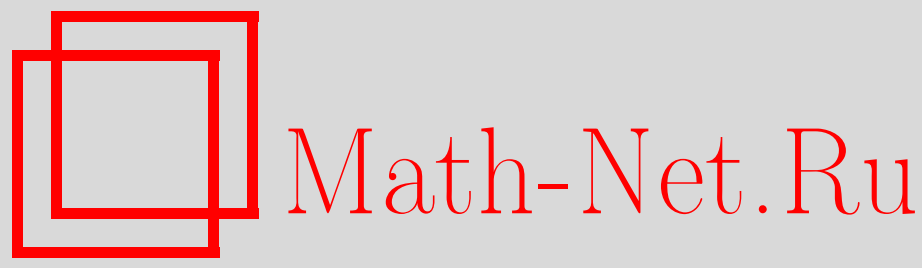

О. Л. Курнявко, И. В. Широков, Построение инвариантных волновых уравнений скалярных частиц на римановых многообразиях с внешними калибровочными полями, ТМФ, 2008, том 156, номер 2, 237-249

DOI: https://doi.org/10.4213/tmf6244

Использование Общероссийского математического портала Math-Net.Ru подразумевает, что вы прочитали и согласны с пользовательским соглашением http://www . mathnet.ru/rus/agreement

Параметры загрузки:

IP : 54.197 .130 .99

26 апреля 2023 г., 13:33:23

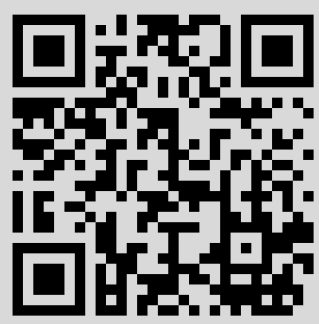




\section{ПОСТРОЕНИЕ ИНВАРИАНТНЫХ ВОЛНОВЫХ УРАВНЕНИЙ СКАЛЯРНЫХ ЧАСТИЦ НА РИМАНОВЫХ МНОГООБРАЗИЯХ С ВНЕШНИМИ КАЛИБРОВОЧНЫМИ ПОЛЯМИ}

Рассмотрена задача построения волновых уравнений для скалярных частиц в римановых пространствах с внешними калибровочными полями, группой симметрии которых является группа движений данного риманова пространства.

Ключевые слова: группа Ли, алгебра Ли, риманово пространство, группа движений, скалярные частицы, уравнение Клейна-Гордона, калибровочные поля.

\section{1. ВВЕДЕНИЕ}

Одной из актуальных задач теоретической физики является интегрирование квантово-полевых уравнений с внешними полями. Известно достаточно большое количество работ, в которых рассматриваются задачи классификации внешних полей, допускающих точное решение квантово-полевых уравнений (см. [1], [2] и цитированную там литературу). Специфика этих работ состоит в том, что преимущественно рассматривается класс уравнений, допускающих реализацию схемы разделения переменных, т.е., как минимум, обладающих коммутативной группой симметрий. Отметим также, что наряду с квантово-полевыми системами активно исследуются аналогичные классические системы (см., например, [3]-[5]).

В работе [6] был предложен метод интегрирования уравнений с некоммутативной группой симметрии. Этот метод дает возможность расширить классификацию полей, допускающих существование точных решений, включив в нее такие поля, наличие которых позволяет рассматривать уравнения, интегрируемые в некоммутативном смысле.

В настоящей работе предлагается метод нахождения внешних (неабелевых в общем случае) полей, наличие которых допускает в качестве группы симметрии уравнения скалярного поля во внешнем калибровочном поле группу движений данного

\footnotetext{
* Омский филиал Института физики полупроводников СО РАН, Омск, Россия. E-mail: kurnyavko@mail.ru

${ }^{\dagger}$ Иртышский филиал Новосибирской государственной академии водного транспорта, Омск, Россия. E-mail: iv_shirokov@mail.ru
} 
пространства. Рассмотренные в работе конструкции позволяют свести решение исходных задач к решению некоторых алгебраических уравнений.

\section{2. ПОСТАНОВКА ЗАДАЧИ}

2.1. Пусть имеется риманово пространство $M$ с метрикой $\mathrm{g}_{a b}=\mathrm{g}_{a b}(x), x \in M$, $a=1, \ldots, \operatorname{dim} M$, допускающее группу движений $G, \mathcal{G}$ - алгебра Ли генераторов действия группы $G$ на $M$, т.е.

$$
\left[X_{i}, X_{j}\right]=C_{i j}^{k} X_{k}, \quad X_{i}=X_{i}^{a}(x) \partial_{a}, \quad X \in \mathcal{G}, \quad x \in M, \quad i=1, \ldots, \operatorname{dim} \mathcal{G} .
$$

Тогда метрика удовлетворяет уравнению Киллинга

$$
\mathcal{L}_{X_{i}} \mathrm{~g}(x)=0
$$

где $\mathcal{L}_{X_{i}} \mathrm{~g}(x)$ - производная Ли вдоль векторного поля $X_{i}$.

Рассмотрим модель скалярного поля, взаимодействующего с калибровочным (неабелевым в общем случае) полем, описываемого лагранжианом

$$
\mathcal{L}=\mathrm{g}^{a b}\left(\nabla_{a}+\overline{\mathcal{A}}_{a}\right) \bar{\psi}\left(\nabla_{b}+\mathcal{A}_{b}\right) \psi-m^{2} \bar{\psi} \psi
$$

где $\psi(x), \bar{\psi}(x)$ - скалярные полевые (в общем случае $n$-компонентные) функции, $\mathcal{A}_{a}-$ калибровочное поле. Этот лагранжиан обладает инвариантностью относительно группы $K$ калибровочных преобразований вида

$$
\begin{aligned}
\psi \rightarrow \psi^{\prime} & =g(x) \psi, & g(x) & \in T(K), \\
\mathcal{A}_{a} \rightarrow \mathcal{A}_{a}^{\prime} & =g(x) \mathcal{A}_{a} g^{-1}(x)-\partial_{a} g(x) g^{-1}(x), & \mathcal{A}_{a} & \in T(\mathcal{K}),
\end{aligned}
$$

где $T(K)$ - некоторое матричное представление группы $K, \mathcal{K}$ и $T(\mathcal{K})$ - алгебра Ли группы $K$ и ее представление, реализованное в виде матриц; при этом полевые функции следует рассматривать как элементы пространства представления алгебры $\mathcal{K}$. Полевые уравнения модели скалярного поля, взаимодействующего с неабелевым калибровочным полем, имеют вид

$$
\begin{gathered}
\left(H+m^{2}\right) \psi(x)=0, \\
H=\mathrm{g}^{a b}(x)\left(\nabla_{a}+\mathcal{A}_{a}\right)\left(\nabla_{b}+\mathcal{A}_{b}\right) .
\end{gathered}
$$

Наличие взаимодействия с калибровочным полем в общем случае приводит к тому, что полевые уравнения теряют имевшиеся симметрии. В связи с этим возникает вопрос о существовании калибровочных полей, наличие которых допускает в качестве группы симметрии уравнения (5) группу движений данного риманова пространства. Очевидно, что искомые калибровочные поля определяются из условия инвариантности оператора $H$ относительно $G$. Заметим, что в этом случае оператор симметрии имеет вид $\widehat{X}=A^{a}(x) \partial_{a}+A(x)$. Тогда получаем следующую систему уравнений, определяющих данные поля:

$$
\begin{gathered}
{\left[H, \widehat{X}_{i}\right]=0} \\
{\left[\widehat{X}_{i}, \widehat{X}_{j}\right]=C_{i j}^{k} \widehat{X}_{k}, \quad \widehat{X}_{i}=X_{i}+\chi_{i}(x),}
\end{gathered}
$$


где $C_{i j}^{k}$ - структурные константы, определяемые в $(1), \chi_{i}$ - функции на $M$, принимающие значения в множестве матриц, действующих в пространстве представления $T(\mathcal{K})$. При калибровочных преобразованиях $(3),(4)$ оператор симметрии преобразуется по закону

$$
\widehat{X}_{i} \rightarrow \widehat{X}_{i}^{\prime}=g \widehat{X}_{i} g^{-1}=X_{i}+\chi_{i}^{\prime}(x)
$$

где $\chi_{i}^{\prime}(x)=X_{i}^{a} g \partial_{a}\left(g^{-1}\right)+g \chi_{i}(x) g^{-1}$. Система уравнений $(7)$ эквивалентна системе

$$
\begin{gathered}
\nabla_{a}\left(X_{i b}\right)+\nabla_{b}\left(X_{i a}\right)=0 \\
\mathrm{~g}^{a b} \nabla_{a}\left(\chi_{i}\right)+\mathrm{g}^{a c} \nabla_{c}\left(X_{i}^{b}\right) \mathcal{A}_{a}-\mathrm{g}^{a b} X_{i}^{c} \nabla_{c}\left(\mathcal{A}_{a}\right)+\mathrm{g}^{a b}\left[\mathcal{A}_{a}, \chi_{i}\right]=0, \\
\mathrm{~g}^{a b} \nabla_{a} \nabla_{b}\left(\chi_{i}\right)-\mathrm{g}^{a b} X_{i}^{c} \nabla_{a, c}^{2}\left(\mathcal{A}_{b}\right)-\mathrm{g}^{a b} X_{i}^{c} \nabla_{c}\left(\mathcal{A}_{a} \mathcal{A}_{b}\right)+2 \mathrm{~g}^{a b} \nabla_{b}\left(\chi_{i}\right) \mathcal{A}_{a}+ \\
+\mathrm{g}^{a b}\left[\nabla_{a}\left(\mathcal{A}_{b}\right), \chi_{i}\right]+\mathrm{g}^{a b}\left[\mathcal{A}_{a} \mathcal{A}_{b}, \chi_{i}\right]=0,
\end{gathered}
$$

где $X_{i a}=\mathrm{g}_{a b} X_{i}^{b}$. Заметим, что система (10) обладает инвариантностью относительно преобразований (4), (9).

2.2. В общем случае система уравнений (10) позволяет решать задачу о классификации римановых пространств, допускающих существование калибровочных полей специального вида, т.е. по заданной группе преобразований некоторого многообразия (точнее по генераторам $X_{i}$ данной группы), действующей транзитивно, необходимо найти функции $\mathrm{g}^{a b}, \chi_{i}, \mathcal{A}_{a}$. Непосредственное интегрирование системы (10) является весьма сложной задачей. Практически некоторое упрощение достигается в силу того, что первое из уравнений данной системы есть уравнение Киллинга, поэтому задача нахождения метрики сводится к классификации римановых пространств по группам движений, полученной в [7]. Однако даже с учетом этого интегрирование оставшихся уравнений является непростой задачей. Настоящая работа посвящена построению общего метода интегрирования системы (10).

2.3. Предлагаемый метод состоит в том, что функции $\mathrm{g}^{a b}$ и $\chi_{i}$ однозначно определяются группой преобразований, действующей на данном однородном пространстве, и могут быть найдены независимо друг от друга, а затем с их помощью определяются калибровочные поля $\mathcal{A}_{a}$.

Функции $\chi_{i}$ могут быть найдены с помощью теории $\mathfrak{g l}(V)$-продолжений, предложенной в [6]. Данная теория предлагает способ построения для заданной алгебры дифференциальных операторов первого порядка $\left\{X_{i}\right\}$ всех возможных алгебр дифференциальных операторов первого порядка $\left\{\widehat{X}_{i}=X_{i}+\chi_{i}\right\}$, удовлетворяющих тем же коммутационным соотношениям (заметим, что подобные объекты представляют собой частный случай так называемых однородных расслоений, общее определение которых можно найти в [8]). Таким образом, для заданной алгебры $\mathcal{G}=\left\{X_{i}\right\}$ могут быть найдены все возможные функции $\chi_{i}$.

В случае, если однородное пространство является группой Ли, задача нахождения функций $\mathrm{g}^{a b}$ и $\mathcal{A}_{a}$ решается относительно просто. В общем случае однородного пространства данная задача может быть сведена в некотором смысле к случаю группы Ли. Используется тот факт, что всякой функции на однородном пространстве $M$ однозначно соответствует некоторая функция из подпространства функционального 
пространства гладких функций на группе $G$, а всякому оператору на $M$ однозначно соответствует оператор из некоторого множества операторов на группе $G$. Если группа действует справа на данном однородном пространстве, то генераторы правых сдвигов на данной группе (левоинвариантные поля) при канонической проекции переходят в генераторы действия группы на данном однородном пространстве, а оператор, инвариантный относительно правых сдвигов, удовлетворяющий некоторому (и что существенно, алгебраическому) дополнительному условию, переходит в оператор на однородном пространстве, инвариантный относительно действия группы. При таком подходе необходимость знания явного вида правоинвариантных полей нисколько не усложняет исходную задачу, так как способы их построения известны. Таким образом, в рассматриваемом подходе построение инвариантных операторов является задачей, решаемой методами линейной алгебры.

В зависимости от того, какое именно дополнительное условие было использовано, из инвариантного оператора общего вида на группе $G$ мы можем получить инвариантный оператор $R_{M}$ на однородном пространстве $M$ либо с условием инвариантности $\left[R_{M}, X_{i}\right]=0$, либо $\left[R_{M}, X_{i}+\chi_{i}(x)\right]=0$. Первому из этих условий могут удовлетворять только дифференциальные операторы, в которых коэффициентами при производных являются скалярные функции. Второму условию в общем случае удовлетворяют операторы с коэффициентами, являющимися матричными функциями. В связи с этим в первом случае мы будем говорить о скалярных инвариантных onepamopax, а во втором о матричных.

Получив таким образом инвариантный оператор вида $R_{M}=\mathrm{g}^{a b}(x) \partial_{a} \partial_{b}+P^{a}(x) \partial_{a}+$ $P(x)$, мы затем докажем, что $\mathrm{g}^{a b}$ - инвариантная метрика на некотором римановом пространстве, а сравнив его вид с (6), получим выражения для $\mathcal{A}_{a}$.

2.4. С методической точки зрения целесообразно сначала рассмотреть задачу построения скалярного инвариантного оператора на данном однородном пространстве, а затем решить аналогичную задачу для матричного инвариантного оператора. В заключение будет приведен пример использования данного метода для построения неабелева калибровочного поля.

\section{3. ПОСТРОЕНИЕ СКАЛЯРНОГО ИНВАРИАНТНОГО ДИФФЕРЕНЦИАЛЬНОГО ОПЕРАТОРА}

3.1. Пусть $M$ - однородное правое $G$-пространство, где $G$ - группа Ли, действующая на пространстве $M, H$ - подгруппа изотропии некоторой точки $x_{0} \in M$, а $\mathcal{G}$ и $\mathcal{H}$ - алгебры Ли групп $G$ и $H$ соответственно, причем $\mathcal{G}=\mathcal{H}+\mathcal{P}, \mathcal{P} \simeq T_{x_{0}} M$, т.е. базис в алгебре Ли $\mathcal{G}$ имеет вид

$$
\left\{e_{i}\right\}=\left\{e_{\alpha}, e_{a}\right\}, \quad i=1, \ldots, \operatorname{dim} \mathcal{G}, \quad a=1, \ldots, \operatorname{dim} M, \quad \alpha=1, \ldots, \operatorname{dim} H,
$$

где $\left\{e_{\alpha}\right\}$ - базис алгебры $\mathcal{H},\left\{e_{a}\right\}$ - базис пространства $\mathcal{P}$. Обозначим $\mathcal{G}^{\mathrm{L}}$ и $\mathcal{G}^{\mathrm{R}}$ алгебры Ли соответственно левоинвариантных и правоинвариантных полей на $G$, реализованные однородными линейными дифференциальными операторами первого порядка, действующими в пространстве $C^{\infty}(G)$, т.е.

$$
\mathcal{G}^{\mathrm{L}}=\left\{\xi_{i}=\left(L_{g}\right)_{*} e_{i}\right\}, \quad \mathcal{G}^{\mathrm{R}}=\left\{\eta_{i}=-\left(R_{g}\right)_{*} e_{i}\right\}, \quad\left[\xi_{i}, \xi_{j}\right]=C_{i j}^{k} \xi_{k}, \quad\left[\eta_{i}, \eta_{j}\right]=C_{i j}^{k} \eta_{k},
$$


где $i, j, k=1, \ldots, \operatorname{dim} G$, причем алгебра Ли правоинвариантных полей $\mathcal{H}^{\mathrm{R}}=\left\{\eta_{\alpha} \in\right.$ $\left.\mathcal{G}^{\mathrm{R}}, \alpha=1, \ldots, \operatorname{dim} H\right\}$ является реализацией алгебры $\mathcal{H}$.

Группа $G$ является главным расслоением над $M$, т.е. $G=G(M=G / H, H, \pi)$. Задавая (локально) гладкое сечение $s: M \rightarrow G$ расслоения $G$, произвольный элемент группы $g \in G$ можно представить в виде $g=h s(x)$. Над областями тривиализации в расслоении $G$ можно ввести координаты прямого произведения

$$
H \times U=\left\{\left(h^{\alpha}, x^{a}\right), a=1, \ldots, \operatorname{dim} M, \alpha=1, \ldots, \operatorname{dim} H\right\},
$$

где $U \subset M$ - область в $M$ (область тривиализации). Тогда имеет место локальное расщепление координат элемента группы на координаты слоя и базы, т.е. $g=$ $\left(h^{\alpha}, x^{a}\right)$.

3.2. Рассмотрим на группе $G$ функциональное пространство $\mathcal{F} \subset C^{\infty}(G)$, определяемое следующим образом:

$$
\mathcal{F}=\left\{\phi \in C^{\infty}(G) \mid \phi(h g)=\phi(g), g \in G, h \in H\right\},
$$

т.е. $\mathcal{F}$ - есть пространство функций на группе, постоянных на слоях главного расслоения $G(M=G / H, H, \pi)$. Для связной группы $G$ определение пространства $\mathcal{F}$ может быть записано эквивалентным образом:

$$
\mathcal{F}=\left\{\phi \in C^{\infty}(G) \mid \eta_{\alpha} \phi(g)=0, g \in G, \eta_{\alpha} \in \mathcal{H}^{\mathrm{R}}, \alpha=1, \ldots, \operatorname{dim} H\right\} .
$$

Каждой функции из заданного функционального пространства соответствует некоторая функция на однородном пространстве из класса $C^{\infty}(M)$, т.е. имеет место изоморфизм $\mathcal{F} \cong C^{\infty}(M)$. Условие постоянства функций из $\mathcal{F}$ на слоях главного расслоения позволяет получить явный вид данного изоморфизма. Действительно, если $\phi(g) \in \mathcal{F}$, то в локальных координатах $(h, x)$ имеем $\phi(h s(x))=\phi\left(h^{\prime} s(x)\right)$, следовательно, $\phi(g)=\phi(h, x)=\phi\left(e_{H}, x\right) \equiv \phi(x)$, т.е. вместо произвольного элемента группы $H$ мы можем выбрать единицу.

Введем на $\mathcal{F}$ пространство линейных операторов $L(\mathcal{F})$. Тогда в соответствии с условием (13) это пространство состоит из линейных операторов $R$, действующих в пространстве $C^{\infty}(G)$ и удовлетворяющих равенству

$$
\left[R, \eta_{\alpha}\right] \phi(g)=0, \quad \text { т.е. }\left.\quad\left[R, \eta_{\alpha}\right]\right|_{\mathcal{F}}=0, \quad \phi(g) \in \mathcal{F}, \quad \eta_{\alpha} \in \mathcal{H}^{\mathrm{R}}, \quad R \in L\left(C^{\infty}(G)\right) .
$$

В силу изоморфизма $\mathcal{F} \cong C^{\infty}(M)$ имеет место изоморфизм $L(\mathcal{F}) \cong L\left(C^{\infty}(M)\right)$. Тогда для каждого оператора из пространства $L(\mathcal{F})$ корректно определяется проекция на однородное пространство, которую обозначим так же, как дифференциал канонической проекции $\pi_{*}$. Таким образом, всякий оператор на $M$ может быть получен из некоторого оператора в пространстве $L(\mathcal{F})$ следующим образом: $R_{M}=\pi_{*} R$, $R \in L(\mathcal{F}), R_{M} \in L\left(C^{\infty}(M)\right)$, причем $\left.\pi_{*} R \approx R\right|_{\mathcal{F}}, R \in L(\mathcal{F})$.

3.3. Из коммутативности левоинвариантных и правоинвариантных полей вытекает, что $\left[\xi_{i}, \eta_{\alpha}\right]=0$. Следовательно, каждое левоинвариантное поле проецируется в поле $X_{i}$ на $M$

$$
X_{i}=\pi_{*} \xi_{i}=\left.\xi_{i}\right|_{\mathcal{F}}, \quad \xi_{i} \in \mathcal{G}^{\mathrm{L}},
$$


являющееся генератором действия группы $G$ на многообразии $M$. Тогда очевидно, что дифференциальный оператор $R_{M} \in L\left(C^{\infty}(M)\right)$, инвариантный относительно действия группы $G$ на $M$, имеет вид

$$
R_{M}=\pi_{*} R, \quad R \in L(\mathcal{F})
$$

где

$$
\left[R, \xi_{i}\right]=0
$$

Рассмотрим в качестве $R \in L(\mathcal{F})$ дифференциальный оператор второго порядка, удовлетворяющий условию (17). Тогда

$$
R=B^{i j} \eta_{i} \eta_{j}+B^{i} \eta_{i}+B
$$

где $B^{i j}, B^{i}$ и $B$ - некоторые действительные постоянные, значения которых определяются из (14). Таким образом, в соответствии с выражением (16) оператор $R$ вида (18) определяет оператор $R_{M}=\left.R\right|_{\mathcal{F}}$, который является скалярным дифференциальным оператором второго порядка, инвариантным относительно действия группы $G$ на $M$. В силу того, что правоинвариантные поля $\eta_{\alpha}$ в координатах $(h, x)$ и базисе (11) имеют вид $\eta_{\alpha}=\eta_{\alpha}^{\beta}(h) \partial_{\beta}$, получаем

$$
R_{M}=\left.\left(B^{i j} \eta_{i} \eta_{j}+B^{i} \eta_{i}+B\right)\right|_{\mathcal{F}}=\left.\left(B^{a b} \eta_{a} \eta_{b}+\widetilde{B}^{a} \eta_{a}+B\right)\right|_{\mathcal{F}}
$$

где $\widetilde{B}^{a}=B^{a}+B^{b \alpha} C_{\alpha b}^{a}$. Тогда без ограничения общности вместо оператора (18) рассмотрим оператор вида

$$
R=B^{a b} \eta_{a} \eta_{b}+B^{a} \eta_{a}+B
$$

Нетрудно видеть, что условие (14), примененное к оператору (19), приводит к системе линейных уравнений относительно переменных $B^{a b}$ и $B^{a}$ вида

$$
\begin{aligned}
B^{a b} C_{a \alpha}^{c}+B^{a c} C_{a \alpha}^{b} & =0, \\
B^{a b} C_{a \alpha}^{\beta} C_{\beta b}^{c}+B^{a} C_{a \alpha}^{c} & =0,
\end{aligned}
$$

где $C_{j k}^{i}$ - структурные константы алгебры Ли группы Ли $G$ относительно базиса $(11), a, b, c=1, \ldots, \operatorname{dim} M, \alpha, \beta=1, \ldots, \operatorname{dim} H, i, j, k=1, \ldots, \operatorname{dim} G$. В результате имеет место следующее утверждение.

УТВЕРЖДЕНИЕ 1. Всякий скалярный линейный дифференциальный оператор второго порядка $R_{M}$, действующий в пространстве $C^{\infty}(M)$ на однородном правом $G$-пространстве $M$ и инвариантный относительно действия группы $G$, имеет вид

$$
R_{M}=\left.\left(B^{a b} \eta_{a} \eta_{b}+B^{a} \eta_{a}\right)\right|_{\mathcal{F}}
$$

причем $B^{a b}$ и $B^{a}$ удовлетворяют системе линейных алгебраических уравнений (20). 
3.4. Пусть $R_{M} \in L\left(C^{\infty}(M)\right)$ есть дифференциальный оператор, инвариантный относительно действия группы $G$ на пространстве $M$, вида

$$
R_{M}=g^{a b}(x) \partial_{a} \partial_{b}+P^{a}(x) \partial_{a},
$$

где $\mathrm{g}^{a b}(x), P^{a}(x)$ - некоторые скалярные функции. По определению оператор $R_{M}$ удовлетворяет условию

$$
\left[R_{M}, X_{i}\right]=0,
$$

где $X_{i}$ - генератор действия $G$ на $M$. Нетрудно видеть, что данное условие приводит к уравнению Киллинга (2) относительно $\mathrm{g}^{a b}(x)$. Тогда $\mathrm{g}^{a b}(x)$ есть инвариантная метрика на пространстве $M$, а само пространство $M$ есть риманово многообразие с группой движений $G$. Согласно утверждению 1 оператор $R_{M}$ определяется выражением (21). Отсюда имеем

$$
\begin{aligned}
R_{M} & =\left.\left(B^{a b} \eta_{a}^{i} \eta_{b}^{j} \partial_{i} \partial_{j}+B^{a b} \eta_{a}^{i} \partial_{i}\left(\eta_{b}^{j}\right) \partial_{j}+B^{a} \eta_{a}^{j} \partial_{j}\right)\right|_{\mathcal{F}}= \\
& =B^{a b} \eta_{a}^{c} \eta_{b}^{d} \partial_{c} \partial_{d}+\left(B^{a b} \eta_{a}^{c} \partial_{c}\left(\eta_{b}^{d}\right)+B^{a} \eta_{a}^{d}\right) \partial_{d},
\end{aligned}
$$

где $i, j=1, \ldots, \operatorname{dim} G, a, b, c, d=1, \ldots, \operatorname{dim} M$. Сравнивая выражения (23) и (22), получим, что метрика $\mathrm{g}^{a b}(x)$ имеет вид

$$
\mathrm{g}^{a b}(x)=B^{c d} \eta_{c}^{a}(h, x) \eta_{d}^{b}(h, x), \quad a, b, c, d=1, \ldots, \operatorname{dim} M,
$$

где $B^{a b}$ - величины, удовлетворяющие системе $(20), \eta_{b}^{a}(h, x)$ - компоненты правоинвариантных полей в координатах $\left(h^{\alpha}, x^{a}\right)$ и относительно базиса $(11)$ алгебры Ли $\mathcal{G}$. Таким образом, приходим к следующему утверждению.

УтВЕРЖДЕНИЕ 2. Всякое правое однородное G-пространство $M$ является римановым многообразием с группой движений $G$ тогда и только тогда, когда существует решение системь (20), при этом инвариантная метрика данного риманова пространства имеет вид (24).

Согласно сформулированным выше утверждениям построение скалярного инвариантного оператора, равно как и построение инвариантной метрики, есть задача выбора величин $B^{a b}$ и $B^{a}$, состоящая в решении системы линейных уравнений $(20)$.

\section{4. ПОСТРОЕНИЕ МАТРИЧНОГО ИНВАРИАНТНОГО ДИФФЕРЕНЦИАЛЬНОГО ОПЕРАТОРА}

4.1. Рассмотрим задачу построения матричного дифференциального оператора, инвариантного относительно группы преобразований, действующей на некотором однородном пространстве. Как и выше, рассмотрим однородное пространство $M=G / H$ и группу $G$, действующую на нем. При этом на данной группе по-прежнему будут использоваться базис (11) и координаты прямого произведения (12). Кроме того, введем еще один необходимый в дальнейшем объект - линейное пространство $V$, являющееся пространством представления $T(\mathcal{K})$, и будем считать, что левоинвариантные и правоинвариантные поля реализованы однородными линейными операторами, действующими в пространстве функций $C^{\infty}(G, V)$, 
т.е. в пространстве функций на $G$, принимающих значение в линейном пространстве $V$ ( $n$-компонентные функции).

Введем функциональное пространство $\widehat{\mathcal{F}}$, определяемое следующим образом:

$$
\widehat{\mathcal{F}}=\left\{\phi(g) \in C^{\infty}(G, V) \mid \phi(h g)=U(h) \phi(g)\right\},
$$

где $U(h)$ - некоторое представление подгруппы изотропии $H$. Инфинитезимальным критерием, определяющим данное пространство, является выражение

$$
\left(\eta_{\alpha}+\Lambda_{\alpha}\right) \phi(g)=0
$$

где $\alpha=1, \ldots, \operatorname{dim} H, \eta_{\alpha}-$ правоинвариантные поля на $G$, образующие алгебру $\mathcal{H}$, $\Lambda_{\alpha}$ - генератор представления алгебры $\mathcal{H}$. Каждой функции из данного функционального пространства соответствует некоторая функция на однородном пространстве из класса $C^{\infty}(M, V)$, т.е. имеет место изоморфизм $\widehat{\mathcal{F}} \cong C^{\infty}(M, V)$. Явный вид данного изоморфизма можно получить следующим образом: $\phi(g)=\phi(h s(x))=$ $U(h) \phi(x)$, где $\phi(s(x)) \equiv \phi(x)$.

Введем на $\widehat{\mathcal{F}}$ пространство линейных операторов $L(\widehat{\mathcal{F}})$. Тогда согласно $(25)$ данное пространство состоит из линейных операторов $R$, действующих в пространстве $C^{\infty}(G)$ и удовлетворяющих условию $\left[R, \eta_{\alpha}+\Lambda_{\alpha}\right] \phi(g)=0$, т.е.

$$
\left.\left[R, \eta_{\alpha}+\Lambda_{\alpha}\right]\right|_{\widehat{\mathcal{F}}}=0, \quad \phi(g) \in \widehat{\mathcal{F}}, \quad \eta_{\alpha} \in \mathcal{H}^{\mathrm{R}}, \quad R \in L\left(C^{\infty}(G)\right) .
$$

Условие (26) равносильно условию $\left.\left[U^{-1}(h) R U(h), \eta_{\alpha}\right]\right|_{\mathcal{F}}=0$. Тогда каждому оператору $R \in L(\widehat{\mathcal{F}})$ соответствует оператор $R_{M}$ на однородном пространстве:

$$
R_{M}=\pi_{*}\left(U^{-1}(h) R U(h)\right)=\left.U^{-1}(h) R U(h)\right|_{\mathcal{F}}, \quad R \in L(\widehat{\mathcal{F}}) .
$$

Заметим, что условию (26) удовлетворяют левоинвариантные векторные поля на $G$, так как $\left[\xi_{i}, \eta_{\alpha}\right]=0$, т.е. оператор $\xi_{i}$, действуя на функцию из $\widehat{\mathcal{F}}$, переводит ее снова в некоторую функцию из $\widehat{\mathcal{F}}$. Нетрудно видеть, что в терминах введенного функционального пространства $\widehat{\mathcal{F}}$ оператор симметрии вида (8) может быть получен следующим образом:

$$
\widehat{X}_{i}=\pi_{*}\left(U^{-1}(h) \xi_{i} U(h)\right)=\left.U^{-1}(h) \xi_{i} U(h)\right|_{\mathcal{F}} .
$$

Тогда матричный дифференциальный оператор $R_{M} \in L\left(C^{\infty}(M)\right)$, инвариантный относительно действия группы $G$ на $M$, имеет вид (27), где $R$ удовлетворяет условию (17). Действительно,

$$
\pi_{*}\left(U^{-1}\left[R, \xi_{i}\right] U\right)=\left[\pi_{*}\left(U^{-1} R U\right), \pi_{*}\left(U^{-1} \xi_{i} U\right)\right]=\left[\pi_{*}\left(U^{-1} R U, \widehat{X}_{i}\right]=0, \quad R \in L(\widehat{\mathcal{F}}) .\right.
$$

4.2. Рассмотрим в качестве $R \in L(\widehat{\mathcal{F}})$ дифференциальный оператор второго порядка, удовлетворяющий условию (17). Тогда

$$
R=B^{i j} \eta_{i} \eta_{j}+B^{i} \eta_{i}+B
$$

где $B^{i j}$ - некоторые действительные постоянные, $B^{i}, B$ - действительные матрицы, значения которых определяются из условия (26). Таким образом, согласно формуле (27) оператор $R$ вида (29) определяет оператор $R_{M}=\left.U^{-1}(h) R U(h)\right|_{\mathcal{F}}$, который 
является матричным дифференциальным оператором второго порядка, инвариантным относительно действия группы $G$ на $M$. Можно показать, что имеет место равенство

$$
R_{M}=\left.U^{-1}(h)\left(B^{i j} \eta_{i} \eta_{j}+B^{i} \eta_{i}+B\right) U(h)\right|_{\mathcal{F}}=\left.U^{-1}(h)\left(B^{a b} \eta_{a} \eta_{b}+\widetilde{B}^{a} \eta_{a}+\widetilde{B}\right) U(h)\right|_{\mathcal{F}},
$$

где

$$
\begin{aligned}
\widetilde{B}^{a} & =B^{a}-2 B^{\alpha a} U(h) \Lambda_{\alpha} U^{-1}(h)+B^{b \alpha} C_{b \alpha}^{a}, \\
\widetilde{B} & =B^{\alpha \beta} U(h) \Lambda_{\alpha} \Lambda_{\beta} U^{-1}(h)-B^{\alpha} U(h) \Lambda_{\alpha} U^{-1}(h)-B^{b \beta} C_{b \beta}^{\alpha} U(h) \Lambda_{\alpha} U^{-1}(h)+B .
\end{aligned}
$$

Тогда без ограничения общности вместо оператора (29) можно рассматривать оператор вида

$$
R=B^{a b} \eta_{a} \eta_{b}+B^{a} \eta_{a}+B
$$

Нетрудно видеть, что условие (26), примененное к оператору (30), приводит к системе линейных уравнений относительно неизвестных $B^{a b}, B^{a}$ и $B$ вида

$$
\begin{gathered}
B^{a b} C_{a \alpha}^{c}+B^{a c} C_{a \alpha}^{b}=0 \\
B^{a b} C_{a \alpha}^{\beta} C_{\beta b}^{c}-2 B^{a c} C_{a \alpha}^{\beta} \Lambda_{\beta}+B^{a} C_{a \alpha}^{c}+\left[B^{c}, \Lambda_{\alpha}\right]=0 \\
-\left(B^{a b} C_{a \alpha}^{\beta} C_{\beta b}^{\gamma}+B^{a} C_{a \alpha}^{\gamma}\right) \Lambda_{\gamma}+\left[B, \Lambda_{\alpha}\right]=0 .
\end{gathered}
$$

В результате можно сформулировать следующее утверждение.

УтВЕРЖДЕНИЕ 3. Всякий матричный линейный дифференциалъный оператор второго порядка $R$, действующий в пространстве $C^{\infty}(M, V)$ на однородном правом $G$-пространстве $M$ и инвариантный относительно действия группы $G$, имеет вид

$$
R_{M}=\left.U^{-1}(h)\left(B^{a b} \eta_{a} \eta_{b}+B^{a} \eta_{a}+B\right) U(h)\right|_{\mathcal{F}},
$$

причем $B^{a b}, B^{a}$ и В удовлетворяют системе матричных линейных алгебраических уравнений (31).

Таким образом, мы показали, что построение матричного инвариантного оператора фактически есть задача нахождения величин $B^{a b}, B^{a}$ и $B$, решаемая методами линейной алгебры.

\section{5. ПОСТРОЕНИЕ КАЛИБРОВОЧНЫХ ПОЛЕЙ}

5.1. Приведенные в разделе 4 построения позволяют решить поставленную исходную задачу, т.е. найти решение системы (10) без ее непосредственного интегрирования. Сформулируем главную в данной работе теорему о существовании искомых метрик и калибровочных полей, на основе которой решается исходная задача.

Теорема. Уравнение Клейна-Гордона (5) на римановом многообразии $M$ с метрикой $\mathrm{g}^{a b}$ и некоторой группой движений $G$ допускает калибровочное поле $\mathcal{A}_{a}$, сохраняющее в качестве группы симметрии данного уравнения группу движений данного пространства, т.е. условия (7) и (8) выполняются тогда и только тогда, когда существует решение системы линейных алгебраических уравнений (31) при 
заданных структурных константах алгебры Ли $\mathcal{G}$ в специальном базисе (11). При этом $\chi_{i}$, инвариантная метрика $\mathrm{g}^{a b}(x)$ и калибровочное поле $\mathcal{A}_{a}$ определяются выражениями

$$
\begin{gathered}
\chi_{i}=\xi_{i}^{\alpha}\left(e_{H}, x\right) \Lambda_{\alpha}, \\
\mathrm{g}^{a b}(x)=B^{c d} \eta_{c}^{a} \eta_{d}^{b}, \\
\mathcal{A}_{a}=\mathrm{g}_{a b} \mathrm{~g}^{b \alpha} \Lambda_{\alpha}+\frac{1}{2} \mathrm{~g}_{a b}\left(\left.\left(B^{c d} \eta_{c}^{i} \partial_{i}\left(\eta_{d}^{b}\right)+B^{c} \eta_{c}^{b}\right)\right|_{h=0}-\frac{1}{\sqrt{\mathrm{g}}} \partial_{c}\left(\sqrt{\mathrm{g} g}{ }^{c b}\right)\right),
\end{gathered}
$$

где $e_{H}$ - единица группъ $H$, являющейся подгруппой изотропии группљ $G, B^{a b}, B^{a}$ и $B$ - величинь, удовлетворяющие системе $(31), \xi_{b}^{a}(h, x)$ u $\eta_{b}^{a}(h, x)$ - компоненты левоинвариантных и правоинвариантных полей соответственно, $\mathrm{g}=\operatorname{det} \mathrm{g}_{a b}$.

ДокАЗАТЕЛЬСтво. Произвольный матричный дифференциальный оператор $R_{M} \in L\left(C^{\infty}(M)\right)$, инвариантный относительно действия группы $G$ на $M$, имеет вид

$$
R_{M}=\mathrm{g}^{a b}(x) \partial_{a} \partial_{b}+P^{a}(x) \partial_{a}+P(x),
$$

где $\mathrm{g}^{a b}(x), P^{a}(x), P(x)$ - некоторые матричные функции. По определению оператор $R_{M}$ удовлетворяет условию

$$
\left[R_{M}, \widehat{X}_{i}\right]=0
$$

где $\widehat{X}_{i}=X_{i}+\chi_{i}$ - оператор симметрии, соответствующий действию $G$ на $M$. Согласно теореме о $\mathfrak{g l}(V)$-продолжениях [9] на всяком однородном пространстве функции $\chi_{i}$ определяются с точностью до калибровочного преобразования формулой (33).

Нетрудно видеть, что условие (37) приводит к уравнению Киллинга (2) относительно $\mathrm{g}^{a b}(x)$, т.е. $\mathrm{g}^{a b}(x)$ - инвариантная метрика на пространстве $M$.

Согласно утверждению 3 произвольный инвариантный матричный дифференциальный оператор имеет вид (32). Отсюда вытекает, что

$$
R_{M}=\left.\left(B^{a b} U^{-1}(h) \eta_{a} U(h) U^{-1}(h) \eta_{b} U(h)+B^{a} U^{-1}(h) \eta_{a} U(h)+U^{-1}(h) B U(h)\right)\right|_{\mathcal{F}},
$$

где $a, b=1, \ldots, \operatorname{dim} M$. Для оператора $U^{-1}(h) \eta_{a} U(h)$ нетрудно получить выражение

$$
U^{-1}(h) \eta_{a} U(h)=\eta_{a}^{b}(h, x) \partial_{b}+\eta_{\alpha}^{\beta}(h, x) U^{-1}(h) \frac{\partial U(h)}{\partial h^{\beta}}+\eta_{a}^{\beta}(h, x) \partial_{\beta}
$$

С помощью условия (25) для производной в последнем выражении имеем

$$
\frac{\partial U(h)}{\partial h^{\beta}}=-\eta_{\beta}^{-1 \alpha}(h, x) \Lambda_{\alpha} U(h) .
$$

В результате получим

$$
U^{-1}(h) \eta_{a} U(h)=\eta_{a}^{b}(h, x) \partial_{b}-\eta_{a}^{\beta}(h, x) \eta_{\beta}^{-1 \alpha}(h, x) U^{-1}(h) \Lambda_{\alpha} U(h)+\eta_{a}^{\beta}(h, x) \partial_{\beta} .
$$

В силу $H$-инвариантности оператора $R_{M}$ после подстановки выражения (39) в формулу (38) в коэффициентах можно положить $h=e_{H}$. Таким образом, компоненты 
инвариантной метрики $\mathrm{g}^{a b}$ имеют вид (34), а $P^{a}$ и $P$ в формуле (36) определяются следующим образом:

$$
\begin{aligned}
P^{a}(x) & =2 \mathrm{~g}^{\alpha a} \Lambda_{\alpha}+\left.\left(B^{c d} \eta_{c}^{i} \partial_{i}\left(\eta_{d}^{a}\right)+B^{c} \eta_{c}^{a}\right)\right|_{h=0}, \\
P(x) & =\mathrm{g}^{\alpha \beta} \Lambda_{\alpha} \Lambda_{\beta}+\left.\left(B^{c d} \eta_{c}^{i} \partial_{i}\left(\eta_{d}^{\alpha}\right) \Lambda_{\alpha}+B^{c} \eta_{c}^{\alpha} \Lambda_{\alpha}\right)\right|_{h=0}+B,
\end{aligned}
$$

где $\mathrm{g}^{\alpha b}=\left.B^{c d} \eta_{c}^{\alpha} \eta_{d}^{b}\right|_{h=0}, \mathrm{~g}^{\alpha \beta}=\left.B^{c d} \eta_{c}^{\alpha} \eta_{d}^{\beta}\right|_{h=0}$. Отметим, что $B^{c d}$ как решения системы (31) обеспечивают независимость величин $\mathrm{g}^{a b}$, являющихся компонентами инвариантной метрики, от переменных $h$.

Очевидно, что произвольный дифференциальный оператор второго порядка всегда может быть приведен к виду

$$
R_{M}=\frac{1}{\sqrt{\mathrm{g}}}\left(\partial_{a}+\mathcal{A}_{a}\right) \sqrt{\mathrm{gg}}^{a b}\left(\partial_{b}+\mathcal{A}_{b}\right)+\varphi,
$$

где

$$
\mathcal{A}_{a}=\frac{1}{2} \mathrm{~g}_{a b}\left[P^{b}-\frac{1}{\sqrt{\mathrm{g}}} \partial_{a}\left(\sqrt{\mathrm{gg}}^{a b}\right)\right], \quad \varphi=P-\frac{1}{\sqrt{\mathrm{g}}} \partial_{a}\left(\sqrt{\mathrm{gg}}^{a b} \mathcal{A}_{b}\right)-\mathrm{g}^{a b} \mathcal{A}_{a} \mathcal{A}_{b}, \quad \mathrm{~g}=\left|\mathrm{g}_{a b}\right| .
$$

Тогда калибровочный потенциал $\mathcal{A}_{a}$ имеет вид (40). Можно показать, что $\varphi$ является постоянной диагональной матрицей и справедливо представление

$$
\varphi=\frac{1}{4}\left(B^{a b} C_{a c}^{c} C_{b d}^{d}-B_{a b} B^{a} B^{b}\right),
$$

которое обеспечивает инвариантность оператора

$$
H=\frac{1}{\sqrt{\mathrm{g}}}\left(\partial_{a}+\mathcal{A}_{a}\right) \sqrt{\mathrm{gg}}^{a b}\left(\partial_{b}+\mathcal{A}_{b}\right), \quad H \in L\left(C^{\infty}(M)\right) .
$$

Таким образом, теорема доказана.

5.2. Приведем общий алгоритм решения поставленной задачи. Пусть дано гладкое вещественное однородное пространство $M=G / H$, где $G$ - вещественная группа Ли, действующая транзитивно на $M, H \subset G$ - подгруппа Ли группы $G$, которая является подгруппой изотропии некоторой точки пространства $M$. Пусть задана группа $G L(m) \supseteq T(K)$, где $K$ - группа калибровочных преобразований. Тогда последовательность действий, составляющих рассматриваемый алгоритм, следующая:

1) строим левоинвариантные и правоинвариантные поля на группе $G$;

2) выбираем некоторое представление подалгебры изотропии $\mathcal{H}$, действующее в пространстве представления $\mathbb{R}^{m}$ алгебры $K$;

3) вычисляем функции $\chi_{i}$ согласно формуле (33);

4) решаем систему (31) и строим инвариантный оператор, удовлетворяющий условию (26);

5) находим инвариантную метрику и калибровочное поле с помощью выражений (34) и (35).

Рассмотрим пример применения данного алгоритма. Пусть $G$ - группа Ли, $\operatorname{dim} G=5$, алгебра Ли которой имеет следующие ненулевые коммутационные соотношения:

$$
\left[e_{1}, e_{5}\right]=e_{1}, \quad\left[e_{2}, e_{4}\right]=e_{1}, \quad\left[e_{3}, e_{4}\right]=e_{2}, \quad\left[e_{3}, e_{5}\right]=-e_{3}, \quad\left[e_{4}, e_{5}\right]=e_{4},
$$


$H$ - подгруппа Ли группы $G, \operatorname{dim} H=2$, алгебра Ли которой имеет базис $e_{4}, e_{5}$, и пусть размерность пространства представления группы калибровочных преобразований $m=2$.

Введем на группе $G$ канонические координаты второго рода, тогда алгебры Ли левоинвариантных и правоинвариантных полей на данной группе соответственно имеют вид

$$
\begin{gathered}
\xi_{1}=\partial_{1}, \quad \xi_{2}=\partial_{2}, \quad \xi_{3}=\partial_{3}, \\
\xi_{4}=x_{2} \partial_{1}+x_{3} \partial_{2}+\partial_{4}, \quad \xi_{5}=x_{1} \partial_{1}-x_{3} \partial_{3}+x_{4} \partial_{4}+\partial_{5} ; \\
\eta_{1}=-e^{x_{5}} \partial_{1}, \quad \eta_{2}=-x_{4} \partial_{1}-\partial_{2}, \quad \eta_{3}=-\frac{1}{2} x_{4}^{2} e^{-x_{5}} \partial_{1}-x_{4} e^{-x_{5}} \partial_{2}-e^{-x_{5}} \partial_{3}, \\
\eta_{4}=-e^{x_{5}} \partial_{4}, \quad \eta_{5}=-\partial_{5} .
\end{gathered}
$$

Напомним, что данные операторы действуют в пространстве $n$-компонентных функций и являются диагональными матрицами (здесь и далее единичные матрицы явно не указываются).

Выберем в качестве двумерного представления подалгебры изотропии $\mathcal{H}$ в пространстве $\mathbb{R}^{2}$ матрицы присоединенного представления данной алгебры, т.е.

$$
T\left(e_{4}\right)=\left(\begin{array}{ll}
0 & 1 \\
0 & 0
\end{array}\right) \equiv \Lambda_{1}, \quad T\left(e_{5}\right)=\left(\begin{array}{cc}
-1 & 0 \\
0 & 0
\end{array}\right) \equiv \Lambda_{2} .
$$

Рассматривая $G$ как расслоение над многообразием $M=G / H$, отождествим $x_{1}, x_{2}$, $x_{3}$ с координатами базы (т.е. с координатами на $M$ ), а $x_{4}, x_{5}-$ с координатами слоя (т.е. с координатами на $H, x_{4} \equiv h_{1}, x_{5} \equiv h_{2}$ ). Генераторы действия группы $G$ на $M$ имеют вид

$$
X_{1}=\partial_{1}, \quad X_{2}=\partial_{2}, \quad X_{3}=\partial_{3}, \quad X_{4}=x_{2} \partial_{1}+x_{3} \partial_{2}, \quad X_{5}=x_{1} \partial_{1}-x_{3} \partial_{3},
$$

a $\mathfrak{g l}(V)$-продолжениями данных операторов являются операторы вида

$$
X_{1}=\partial_{1}, \quad X_{2}=\partial_{2}, \quad X_{3}=\partial_{3}, \quad X_{4}=x_{2} \partial_{1}+x_{3} \partial_{2}+\Lambda_{1}, \quad X_{5}=x_{1} \partial_{1}-x_{3} \partial_{3}+\Lambda_{2} .
$$

Решая систему (31), получаем

$$
\begin{array}{cl}
\left(G^{a b}\right)=a(\operatorname{diag}(1,-1,1))^{\mathrm{T}}, & G^{1}=-b^{2} \sigma_{3}, \\
G^{2}=b^{2} \sigma_{2}, \quad G^{3}=-2 b^{2} \sigma_{1}, & G=c \operatorname{diag}(1,1),
\end{array}
$$

где $a, b, c$ - некоторые действительные постоянные, а матрицы

$$
\sigma_{1}=\left[\begin{array}{ll}
0 & 1 \\
0 & 0
\end{array}\right], \quad \sigma_{2}=\left[\begin{array}{cc}
1 & 0 \\
0 & -1
\end{array}\right], \quad \sigma_{3}=\left[\begin{array}{cc}
0 & 0 \\
-1 & 0
\end{array}\right]
$$

образуют базис двумерного представления алгебры $s l(2)$. Отсюда получаем метрику и потенциал калибровочного поля:

$$
\begin{gathered}
d s^{2}=a\left(2 d x^{1} d x^{3}-\left(d x^{2}\right)^{2}\right), \\
A_{1}=\frac{b^{2}}{a} \sigma_{1}, \quad A_{2}=\frac{b^{2}}{2 a} \sigma_{2}, \quad A_{3}=\frac{b^{2}}{2 a} \sigma_{3} .
\end{gathered}
$$


Форма напряженности данного калибровочного поля имеет вид

$$
F=-\frac{b^{4}}{a^{2}} \sigma_{1} d x^{1} \wedge d x^{2}-\frac{b^{4}}{2 a^{2}} \sigma_{2} d x^{1} \wedge d x^{3}-\frac{b^{4}}{2 a^{2}} \sigma_{3} d x^{2} \wedge d x^{3} .
$$

\section{6. ЗАКЛЮЧЕНИЕ}

Предложенный в работе подход позволяет с помощью методов линейной алгебры решать задачу о построении на однородных римановых многообразиях релятивистских волновых уравнений (в данном случае рассматривалось уравнение Клейна-Гордона) с калибровочными полями (неабелевыми в общем случае) такими, что группой симметрии полученных уравнений является группа движений данного пространства.

Условие интегрируемости данных уравнений на однородном римановом многообразии совпадает с условием интегрируемости геодезического потока с $G$-инвариантной метрикой на однородном многообразии $M$ и имеет вид $d(M)<2[10]$, где $d(M)-$ дефект данного многообразия (в частности, для симметрических и слабосимметрических пространств $d(M)=0)$.

Авторами была проделана работа по классификации римановых пространств и калибровочных полей, допускающих в качестве группы симметрии уравнения Клейна-Гордона группу движений данного пространства для случая четырехмерных пространств с пятимерными группами движения. В каждом из этих случаев уравнение Клейна-Гордона является интегрируемым. В общей сложности найдено двадцать два пространства с абелевыми калибровочными полями. Авторы предполагают опубликовать полученную классификацию в ближайшее время.

\section{Список литературы}

[1] В. Г. Багров, Д. М. Гитман, И. М. Тернов и др., Точные решения релятивистских волновых уравнений, Наука, Новосибирск, 1982.

[2] V. G. Bagrov, D. M. Gitman, Exact Solutions of Relativistic Wave Equations, Math. Appl., 39, Kluwer, Dordecht etc., 1990.

[3] Д. И. Ефимов, Сиб. матем. журн., 45:3 (2004), 566-576.

[4] Д. И. Ефимов, Сиб. матем. журн., 46:1 (2005), 106-118.

[5] A. V. Bolsinov, B. Jovanovic, J. Phys. A, 39:16 (2006), L247-L252; arXiv: math-ph/0602016.

[6] А. В. Шаповалов, И. В. Широков, ТМФ, 104:2 (1995), 195-213.

[7] А. З. Петров, Пространства Эйнштейна, Физматлит, М., 1961.

[8] В. В. Горбацевич, А. Л. Онищик, "Группы Ли преобразований", Группы Ли и алгебры Лu, 1, Современные проблемы математики. Фундаментальные направления. Итоги науки и техники, 20, ред. Р. В. Гамкрелидзе, ВИНИТИ АН СССР, М., 1988, 103-240.

[9] С. П. Барановский, И. В. Широков, ТМФ, 135:1 (2003), 70-81.

[10] А. А. Магазев, И. В. Широков, ТМФ, 136:3 (2003), 365-379.

Поступила в редакцию 19.07.2007, после доработки 17.10.2007 